\title{
X-Ray Detected Magnetic Hysteresis of Thermally Evaporated Terbium Double-Decker Oriented Films
}

\author{
By Ludovica Margheriti, Daniele Chiappe, Matteo Mannini,* Pierre-E. Car, \\ Philippe Sainctavit, Marie-Anne Arrio, Francesco Buatier de Mongeot, Julio C. Cezar, \\ Federica M. Piras, Agnese Magnani, Edwige Otero, Andrea Caneschi, and Roberta Sessoli*
}

Fabrication of molecular nanostructures and control of the molecular properties at the nanoscale is at the basis of the development of innovative single molecule devices. ${ }^{[1]}$ Particularly active is the research for the organization of single molecule magnets (SMMs) that have been proposed as ideal candidates for the development of molecular spintronics and data storage devices. ${ }^{[2,3]}$ These molecules are a well known class of compounds characterized by the peculiar presence of a strong axial magnetic anisotropy that induces a slow relaxation in the magnetization and a magnetic hysteresis of molecular origin showing spectacular quantum effects. ${ }^{[4]}$ Thanks to the surface sensitivity of synchrotron-based techniques it has been possible to provide the proof of concept that SMM behavior is observable in a single layer of magnetic molecules. ${ }^{[5]}$ First attempts to control at the nanoscale the SMM assembling have been made by opportune functionalization promoting their grafting on specific surfaces in order to form monolayer deposits from solution. ${ }^{[6,7]}$ However, cleaner processes, e.g. thermal evaporation, ${ }^{[8]}$ are required for the development of real devices or to extend the investigation to reactive surfaces, e.g. ferromagnetic metals, and

[*] L. Margheriti, Dr. M. Mannini, Dr. P.-E. Car, Prof. A. Caneschi, Prof. R. Sessoli Department of Chemistry

University of Firenze

INSTM Research Unit and ISTM-CNR

V. della Lastruccia, 3, 50019, Sesto Fiorentino, (FI) (Italy)

E-mail:matteo.mannini@unifi.it; roberta.sessoli@unifi.it

D. Chiappe, Prof. F. B. de Mongeot

Department of Physics

University of Genova and CNISM

V. Dodecaneso, 33, 16146, Genova (Italy)

Prof. Ph. Sainctavit, Dr. M.-A. Arrio

Institut de Minéralogie et de Physique des Milieux Condensés, UMR7590

Université Pierre et Marie Curie

Case 115, 4 place Jussieu, 75252 Paris Cedex 5 (France)

Dr. J. C. Cezar

European Synchrotron Radiation Facility (ESRF)

38043 Grenoble (France)

Dr. F. M. Piras, Prof. A. Magnani

Department of Pharmaceutical and Applied Chemistry

University of Siena

V. A. Moro, 2, 53100, Siena (Italy)

Dr. E. Otero

Synchrotron Soleil

Saint Aubin, 91192 Gif sur Yvette (France) this can be achieved only by ultrahigh vacuum (UHV)-based techniques.

Successful attempts to deposit SMMs in UHV conditions have been carried out with a tetranuclear iron(III) cluster ${ }^{[9]}$ and lanthanide bis-phthalocyaninato $\left(\mathrm{LnPc}_{2}\right)$ complexes. ${ }^{[10,11]}$ For the latter the seminal works of Ishikawa et al. ${ }^{[12]}$ showed that slow dynamics of the magnetization in these mononuclear complexes is originated directly from their strong single ion anisotropy. Very recently a structural and magnetic characterization of a sub-monolayer deposit $\mathrm{TbPc}_{2}$ based on synchrotronlight techniques has confirmed that this magnetic anisotropy is retained on surface. ${ }^{[13]}$ The absence of the observation of typical slow dynamics of the magnetization has been attributed to the long time-scale of the X-ray based experiments.

We report here a synchrotron-based investigation on neutral $\mathrm{TbPc}_{2}$ evaporated thick and thin films evidencing that the thick film of $\mathrm{TbPc}_{2}$ is characterized by slow relaxation of the magnetization and opening of a butterfly hysteresis cycle at temperatures as high as $15 \mathrm{~K}$ but not observed for the thin film. These differences are accompanied with a drastic change in the orientation of the $\mathrm{TbPc}_{2}$ molecules in the two films.

The neutral bis(phthalocyaninato)terbium complex (Figure 1a) of formula $\mathrm{Tb}\left(\mathrm{C}_{32} \mathrm{H}_{16} \mathrm{~N}_{8}\right)_{2} \cdot \mathrm{CH}_{2} \mathrm{Cl}_{2}$, here abbreviated in $\mathrm{TbPc}_{2}$, was synthesized according to literature procedures, ${ }^{[11,14]}$ and found to have the crystal unit cell and the UV-vis spectrum in agreement with previous reports (see Supporting Information, SI).

$\mathrm{TbPc}_{2}$ microcrystalline powder was then evaporated at $500{ }^{\circ} \mathrm{C}$ on $\mathrm{Al}$ foil in a high vacuum chamber producing a $200 \mathrm{~nm}$ thick film and the chemical composition of the evaporated phase was verified by time-of-flight secondary ion mass spectrometry (ToF-SIMS). The positive ion spectrum of the evaporated film was compared with that of $\mathrm{TbPc}_{2}$ powder pressed down on an Indium foil (Figure 1c). In the high mass region $(m / z 600-1500)$ of both spectra, peaks at $m / z 1183$ and $m / z$ 671 have been detected and assigned to the molecular ion $\left(\mathrm{M}^{+}\right)$ and to the molecular ion fragment generated by loss of one Pc molecule $\left([\mathrm{Tb}+\mathrm{Pc}]^{+}\right)$, respectively. The similarities of the fragmentation pattern of the two spectra indicate that despite the thermal treatment the evaporated molecules retain their molecular integrity. Moreover the peak at $m / z 1183$ assigned to the molecular ion $\left(\mathrm{M}^{+}\right)$shows the isotopic distribution in complete agreement with the theoretical isotopic pattern expected for the $\mathrm{TbPc}_{2}$ molecule (Figure $1 \mathrm{~b}$ ). These results are confirmed by the UV-vis spectra although, as previously reported, ${ }^{[15,16]}$ a relevant broadening of the spectral features upon the evaporation is observed (see SI). 

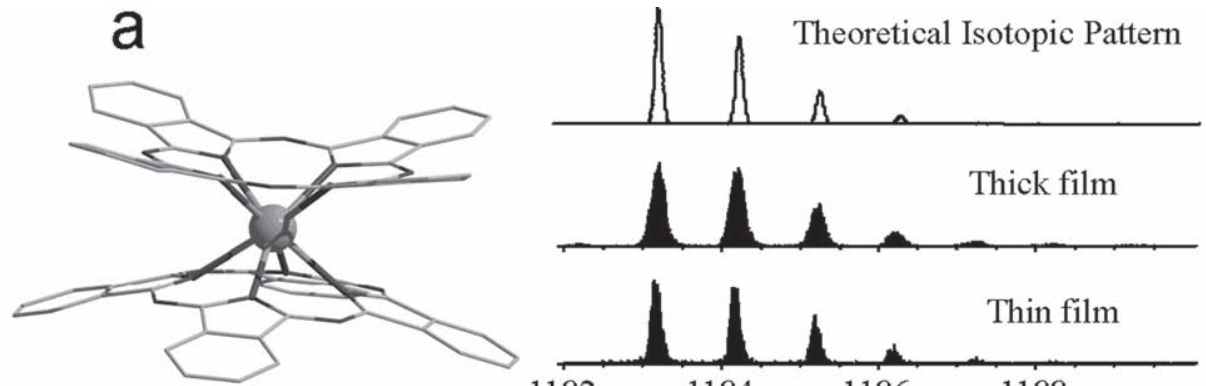

b
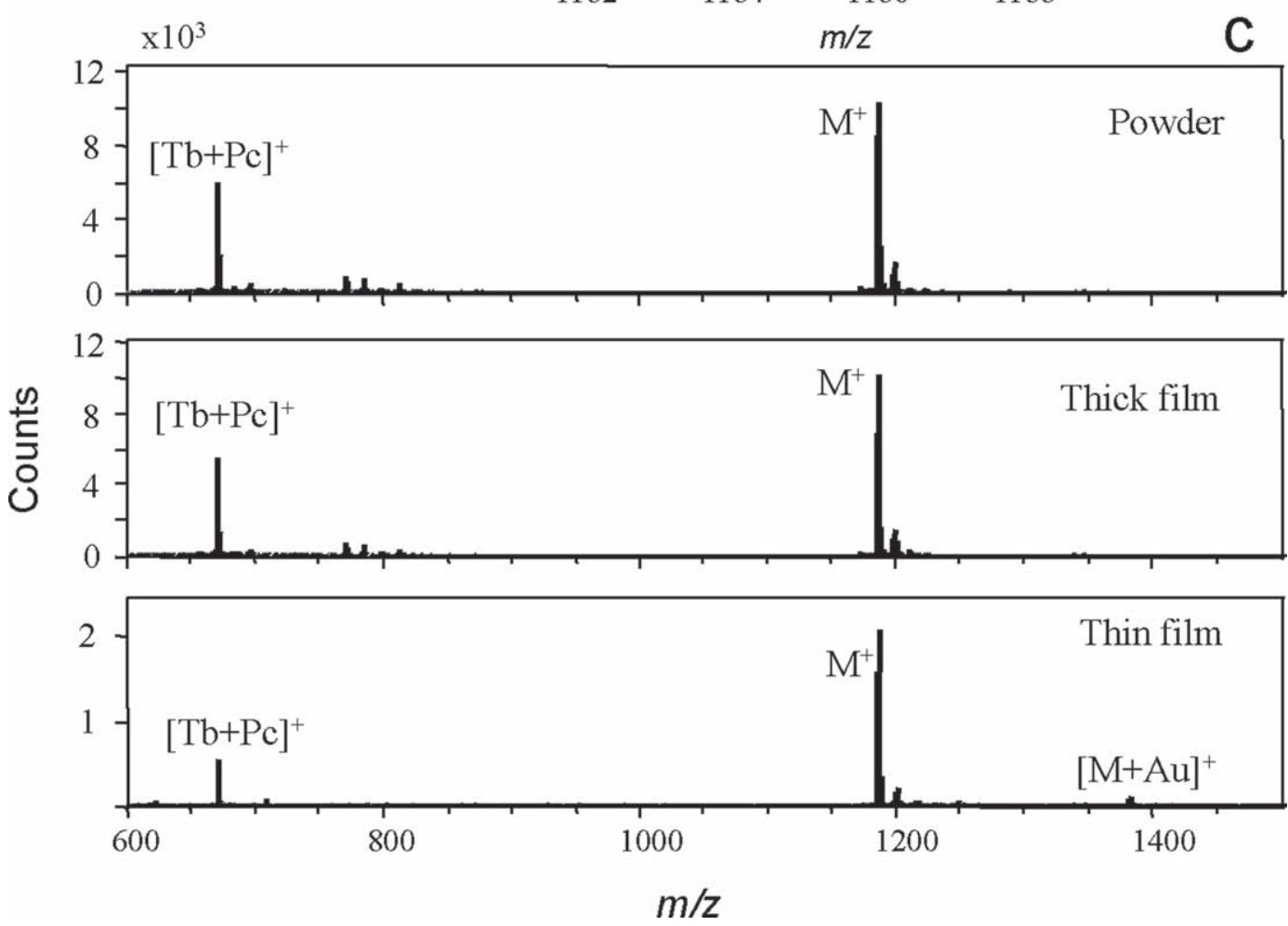

Figure 1. a) Structure of the $\mathrm{TbPc}_{2}$ complex (terbium, grey sphere, nitrogen black stick, carbon light grey stick); b) ToF-SIMS spectra of the evaporated thick and thin film in the molecular ion mass region and the theoretical isotopic distribution; c) Positive ToF-SIMS spectra of the TbPc $\mathrm{C}_{2}$ powder on In (top), $\mathrm{TbPc}_{2}$ thick film evaporated on $\mathrm{Al}$ (middle) and $\mathrm{TbPc}_{2}$ thin film evaporated on Au (bottom) in the mass region between $\mathrm{m} / \mathrm{z} 600-1500$.

The evaporation procedure was repeated on a polycrystalline $\mathrm{Au}$ substrate reducing the exposition time to the monolayer range. The high surface sensitivity of the ToF-SIMS technique allowed the investigation of this thin film. The positive ions spectrum (Figure 1c) coincides with that observed for the thick film with the additional signal at $m / z 1380$ attributable to the $[\mathrm{M}+\mathrm{Au}]^{+}$ion, confirming the monolayer thickness of the deposit. More details relative to the lower mass region $(\mathrm{m} / \mathrm{z}$ $0-600)$ and higher mass region $(m / z 500-2500)$ for all investigated samples are reported in SI.

Structural and magnetic information on the $200 \mathrm{~nm}$ thick film of $\mathrm{TbPc}_{2}$ has been obtained using a variable temperature X-ray absorption spectroscopy (XAS) recorded at the ID08 beamline of the European Synchrotron Radiation Facility (ESRF) in Grenoble. The linear and circularly polarized XAS spectra recorded at $M_{4,5}$ edge of $\mathrm{Tb}$ are presented in Figure $2 \mathrm{a}$ and Figure $2 \mathrm{~d}$. The $3 d$ to $4 f$ transition is strongly dichroic and contains information about the orientation of the molecules, and the magnetic moment of the $4 f$ orbitals. ${ }^{[17]}$ X-ray natural linear dichroism, XNLD, defined as the difference between vertically, $\sigma^{\mathrm{V}}$, and horizontally, $\sigma^{\mathrm{H}}$, polarized light has been measured with the normal to the surface rotated by an angle $\theta=45^{\circ}$ from the photon propagation vector (see inset of Figure 2f). The presence of a relevant dichroic signal (Figure 2a) evidences that the molecules have a preferential orientation in the film. The same kind of investigation, performed on a thinner deposit of $\mathrm{TbPc}_{2}$, of the order of a monolayer, obtained by in situ evaporation on a clean $\mathrm{Au}(111)$ single crystal under UHV condition, lead up to different conclusions. In fact the dichroic signal of the XNLD spectra (Figure 2b), carried out in the same conditions of the thick film, has opposite sign and the intensity is twice larger than that obtained for the evaporated thick film.

In order to clarify the relationship between the detected linear dichroism and the orientation of the molecules we have performed ligand field multiplet (LFM) calculations, ${ }^{[18]}$ following Goedkoop's seminal paper. ${ }^{[17]}$ The spectra of the $M_{4,5}$ edges are 

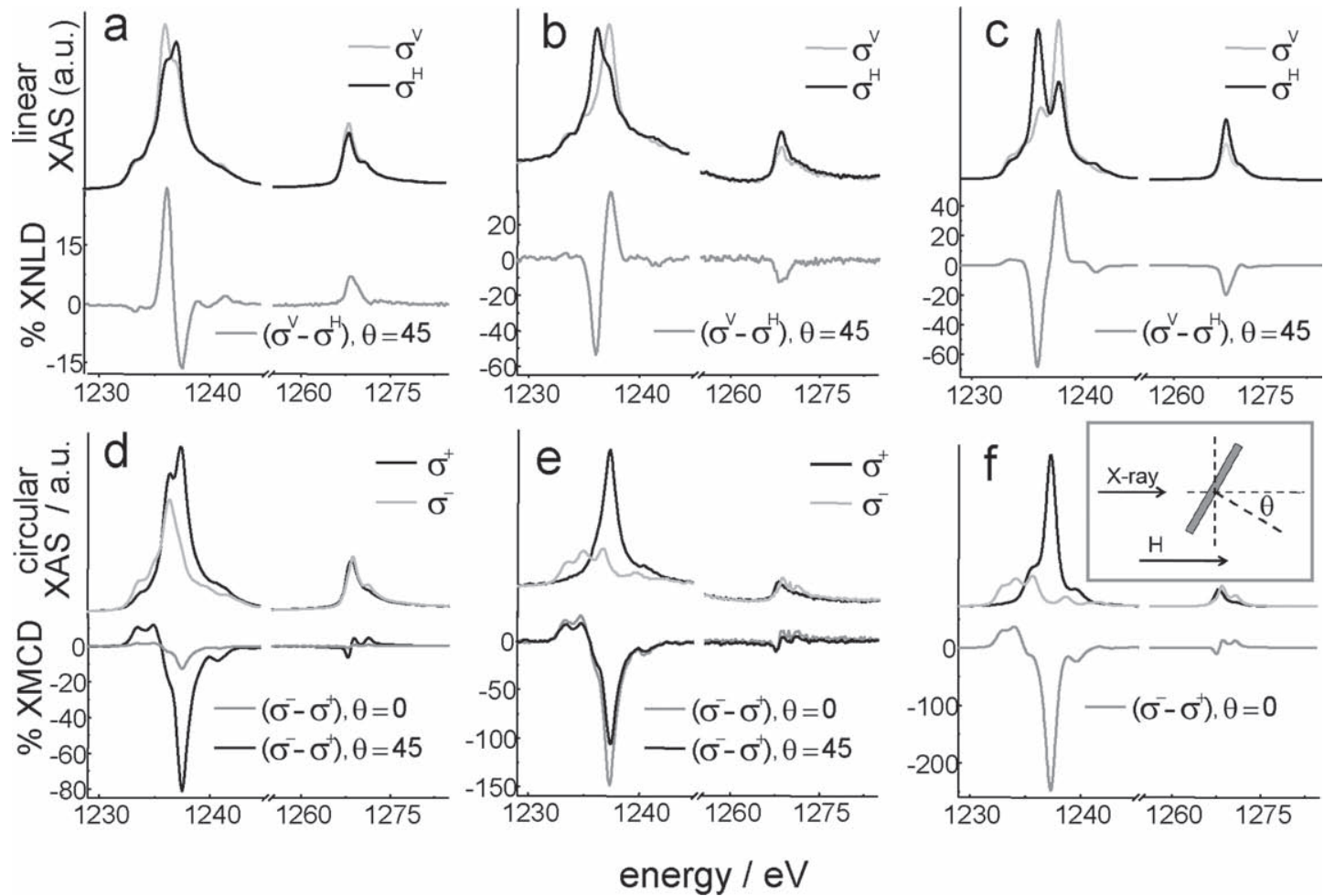

Figure 2. In the upper part: X-ray Absorption Spectra obtained with linearly polarized horizontal, $\sigma^{\mathrm{H}}$, and vertical, $\sigma^{\mathrm{V}}$, light and the normalized dichroic component recorded at $\theta=45^{\circ}, T=8 \mathrm{~K}$ and $B=0 \mathrm{~T}$ for the thick film (a) and the thin film (b) compared to the simulated spectra for $|g s\rangle=\mid \mathrm{J}=6(L=$ $3, S=3), M_{J}= \pm 6>$ (c). In the lower part: left, $\sigma^{+}$, and right, $\sigma^{-}$, circular light XAS and XMCD spectra recorded for the thick film (d) and thin film (e) at $T=8 \mathrm{~K}$ and $B=3 \mathrm{~T}$, at two different angles $\theta$ between the propagation vector and the normal to the substrate. In $\mathrm{f}$ ) the simulated spectra are reported for $|g s\rangle=\mid J=6(L=3, S=3), M_{J}=-6>$. Dichroic percentage contribution are normalized to the isotropic signal at its maximum. In the inset: vertical view of the experimental set up and definition of the $\theta$ angle.

obtained by calculating the electric dipole transitions between the $3 d^{10} 4 f^{8}$ ground state configuration and the $3 d^{9} 4 f^{9}$ excited state configuration. For $\mathrm{Tb}^{3+}$ the ground state of the initial configuration is defined by the following quantum numbers: $L=$ $3, S=3$ and $J=6$, while the first excited state is at too high energy to mix into the ground state. The crystal field, as well as the external magnetic field, lifts the 13-fold degeneracy of the $J=6$ ground state. With these assumptions it has been shown that the $M_{4,5}$ edges depend on geometrical factors and averaged values of the total kinetic momentum operator J acting on the ground state. ${ }^{[19]}$

Assuming that the $\mathrm{TbPc}_{2}$ molecules lie down on the surface with their pseudo-tetragonal, $z$, axis parallel to the normal of the substrate, the intensity of the XNLD signal can be written as $\sigma^{\mathrm{V}}-\sigma^{\mathrm{H}}=1 / 2\left(\sigma \perp-\sigma^{/ /}\right)$, with $\sigma_{/ /}$and $\sigma \perp$ defined as the theoretical cross section measured with the polarization vector parallel and perpendicular to the $\mathrm{z}$ molecular axis. In the case of molecules standing on the surface with the $\mathrm{z}$ axes randomly distributed in the plane of the surface the relation $\sigma^{\mathrm{V}}-\sigma^{\mathrm{H}}=$ $-1 / 4\left(\sigma \perp-\sigma^{/ /}\right)$holds. Having observed a halved and reversed intensity of the linear dichroic contribution in the thick film we can deduce that the molecules are in the standing configuration, while they are lying down in the monolayer.

These ordering effects are not unusual when flat molecules like phthalocyanines or porphyrins are evaporated, because the strong interactions with the substrate, as well as within the molecules, can affect the orientation and packing of the molecular deposit. ${ }^{[20]}$ In particular in MPc systems the first layers grow with the molecule in the "lying" position on noble metal surfaces because of strong molecule-surface interactions, while the molecules adopt a "standing" configuration in the case of rough surfaces and metal-oxide surfaces where moleculemolecule interactions are dominant. ${ }^{[20-24]}$

Our hypothesis is confirmed by the good agreement between the structure of the XNLD signal observed for the thin film and the simulated one (see Figure 2c) assuming lying down configuration. In addition, from a quantitative comparison between the experimental and calculated isotropic spectra and linear dichroic signals, one can determine the averaged value $<$ gs $\left|\left(J_{z}\right)^{2}\right|$ gs $>$ for the molecules, where gs stays for ground state. The extracted value of $30.5 \pm 1$ (in units of $\mathrm{h}^{2}$ ) is close to the limiting value expected for a ground doublet corresponding to pure eigenstates of $J_{z}$ with $M_{J}= \pm 6$, i.e., 36. This result is in agreement with the analysis performed $\mathrm{in}^{[13]}$ and confirms that the tetragonal symmetry axis of $\mathrm{TbPc}_{2}$ is the easy axis of magnetization although a small contribution from other eigenstates of $J_{z}$ or from a non perfect organization of the molecules can be present.

Since $\mathrm{TbPc}_{2}$ molecules are characterized by an easy magnetization axis lying perpendicular to the Pc macrocycles, the 
angular dependence of their magnetic response can also be exploited to confirm the XNLD data and investigate further the organization of the molecules on surface. The X-ray Magnetic Circular Dichroism, XMCD, defined as the difference between the XAS obtained using right $\sigma$ and left $\sigma^{+}$circularly polarized X-rays, has therefore been measured (see SI). The XMCD signal of the evaporated thick film measured at $8 \mathrm{~K}$ with a $3 \mathrm{~T}$ magnetic field normal to the substrate is significantly weaker than the one measured at $\theta=45^{\circ}$, see Figure $2 \mathrm{~d}$, indicating that Tbdouble-decker molecules are arranged in the standing configuration with the easy magnetization axes of the molecules lying in the film plane. On the contrary, in the thin film the strongest XMCD signal is measured with the photon beam normal to the film (see Figure 2e) confirming that the evaporated molecules are arranged in lying configuration with the easy axis of magnetization perpendicular to the surface as already reported. ${ }^{[13]}$ It is interesting to notice the weak XMCD signal detected at $\theta=0^{\circ}$ for the thick film, where the magnetic field is applied along the hard axis of magnetization of the $\mathrm{TbPc}_{2}$ molecules. The normalized intensity observed at $\theta=45^{\circ}$ is significantly smaller than that observed in the thin film at the same angle. In the former case, in fact, at $\theta=45^{\circ}$ there are still molecules with their hard plane parallel to the field due to the random orientation of the molecular axes in the plane of the thick film.

The shape of the XMCD spectra can be nicely reproduced by the LFM calculations introduced above, as shown in Figure $2 \mathrm{f}$ for a lying down configuration. The normalized intensity observed at $T=8 \mathrm{~K}, B=3 \mathrm{~T}$, and $\theta=0^{\circ}$ for the thin film is found to correspond to ca. $60 \%$ of the saturation value, i.e., $T=$ $0 \mathrm{~K}$, expected for a ground state characterized by $M_{J}=-6$.
In order to characterize the SMM behavior of $\mathrm{TbPc}_{2}$ in the two types of deposit, magnetic hysteresis loops at different temperatures and sample orientation have been obtained by monitoring the XMCD signal at $M_{5}$ edge as a function of the applied magnetic field swept at ca. $8 \mathrm{mT} \mathrm{s}^{-1}$. The temperature dependence of the hysteresis loops obtained for the thick film sample (Figure 3a) shows that the evaporated film of $\mathrm{TbPc}_{2}$ has a blocking temperature higher than most SMMs, although with a strong tunneling near zero field that cancels the remnant magnetization and gives a butterfly shape to the hysteresis. Recent magneto-optical investigations of frozen solutions of similar compounds ${ }^{[25]}$ showed butterfly-shape hysteresis for the anionic and cationic derivatives, but not for the neutral one. As our UV-vis spectra exclude that the $\mathrm{TbPc}_{2}$ has undergone a redox reaction during the evaporation, we ascribe the difference between the evaporated film and the frozen solution to packing effects of the molecules. Butterfly shape hysteresis loops observable up to $T=15 \mathrm{~K}$ indeed characterize also the pristine crystalline material.

The angular dependence of the hysteresis (Figure $3 \mathrm{~b}$ ) shows a larger magnitude of the magnetization on increasing the angle that the magnetic field forms with the normal to the surface, thus confirming our previous assumption about the orientation of the molecules.

The thin film was investigated in situ exactly under the same conditions adopted for the thicker film, and the hysteresis loop recorded at $T=8 \mathrm{~K}$ is shown in Figure 4. Interestingly, it was impossible to detect opening of the hysteresis cycle. To explore if slow dynamics of the magnetization is present at lower temperatures we repeated the characterization at the SIM beamline

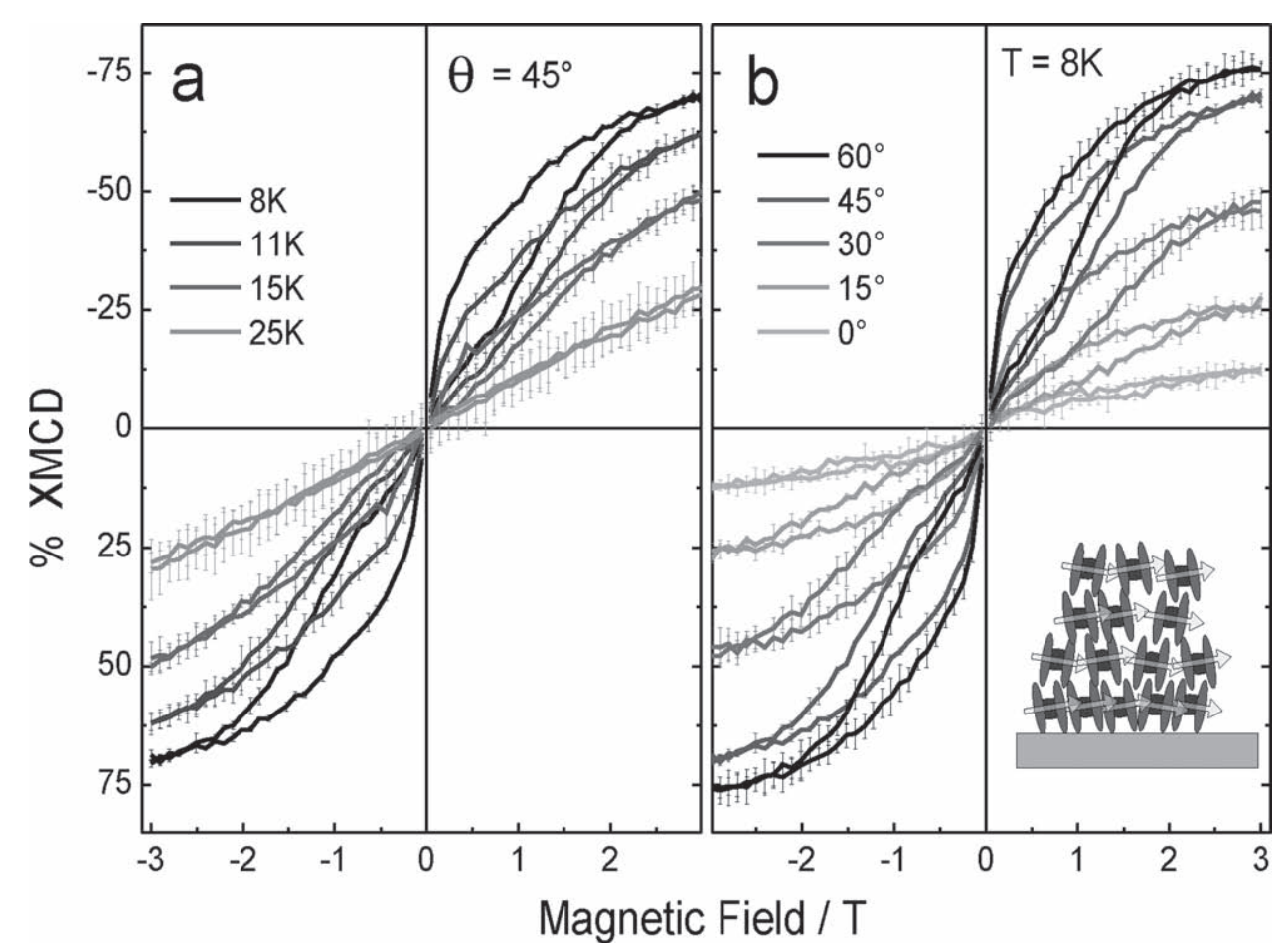

Figure 3. Temperature (a) and angular dependence (b) of the hysteresis loops obtained from the XMCD signal at $1237 \mathrm{eV}$ expressed as percentage of the isotropic XAS. In the inset scheme of the orientation of $\mathrm{TbPc}_{2}$ molecules and their easy axes of magnetization in the thick film. 


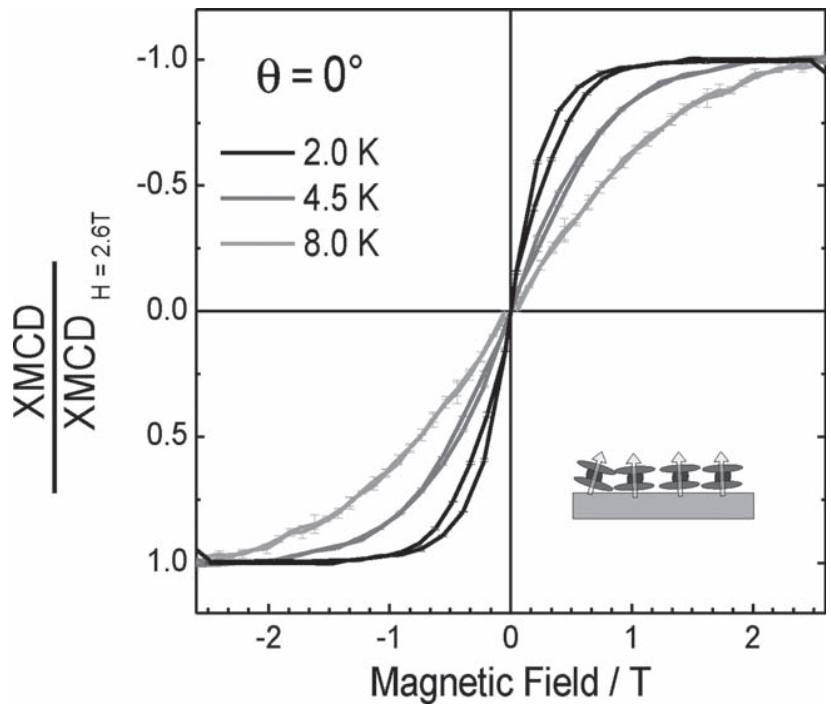

Figure 4. Temperature dependence of the hysteresis loops obtained from the XMCD signal at $1237 \mathrm{eV}$, normalized to the intensity observed at $B=$ 2.6 $\mathrm{T}$. In the inset scheme of the orientation of $\mathrm{TbPc}_{2}$ molecules and their easy axes of magnetization in the thin film.

of the swiss light source (SLS) in Villigen, Switzerland, using the TBT setup for lower temperature measurements. ${ }^{[26]} \mathrm{A}$ similar in situ evaporation of a $\mathrm{TbPc}_{2}$ thin film was this time carried out on a Au polycrystalline film as the lower temperature apparatus does not allow the single crystal treatment properly. The results we obtained at 4.5 and $2.0 \mathrm{~K}$ with normal incidence and sweeping rate of ca. $4 \mathrm{mT} \mathrm{s}^{-1}$ are also reported in Figure 4. The fast saturation at the lowest temperature confirms the preferential orientation of the molecules on surface in lying configuration in agreement with the XNLD and XMCD results reported above. Only a very small opening of the loop and at much lower temperatures compared to the thick film is observed.

At this stage we can exclude that the differences in the magnetic behavior are due to major structural modifications induced by the interaction with the surface, because ToFSIMS analysis of the thin film revealed the presence of intact molecules. The identical XMCD line shape in thick and thin films does not evidence any strong electronic effect induced by the surface proximity, but we underline that the lanthanide edge is not very sensitive to the environment and the occurrence of an interaction between the surface and the Pc ligands cannot be excluded. One could argue that the magnetic irreversibility at relatively high temperature is originated by intermolecular magnetic interactions promoted by the stacked arrangement of the molecules that is not present in the monolayer. However, preliminary measurements on bulk samples of $\mathrm{TbPc}_{2}$ magnetically diluted in $\mathrm{YPc}_{2}$ suggest a more complex scenario. As this investigation involves a temperature region where the magnetic relaxation occurs through a thermally activated process, we should also consider the possibility of a different phonon efficiency in the thick and the thin films, but further investigations are necessary to clarify this point.

To conclude, our magnetic characterization of evaporated $\mathrm{TbPc}_{2}$ molecules carried out on both thick and thin evaporated films confirms that the double decker structure and the peculiar SMM behavior is retained after thermal deposition. Interestingly, this process induces an oriented arrangement of the molecules with the easy axis of magnetization lying in the plane or out of plane depending on the thickness of the film. The spontaneous orientation of molecules, combined with the relatively high temperature opening of the hysteresis loop in the thick film, make Tb double-decker compounds promising candidates for the investigation of the interplay of SMM behavior and transport processes in molecular spintronic devices.

However, it is important to underline that, in contrast to tetranuclear iron(III) complexes that preserve unaltered SMM behavior in the monolayer deposit, ${ }^{[5]} \mathrm{TbPc}_{2}$ shows significant changes in the magnetization dynamics when the thickness is scaled down to the monolayer regime.

\section{Experimental Section}

Synthesis of $\mathrm{TbPC}_{2} \cdot \mathrm{CH}_{2} \mathrm{Cl}_{2}$ : The neutral bis(phthalocyaninato)terbium complex, was synthesized according to the first procedure of Weiss et al. ${ }^{[14]}$ with a slight modification of the heating time, as recently reported elsewhere..$^{[1]}$

Thermal deposition of $\mathrm{TbPc}_{2}$ : A $200 \mathrm{~nm}$ thick film of $\mathrm{TbPc}_{2}$ compound was thermally evaporated from a quartz crucible filled with $30 \mathrm{mg}$ of microcrystalline powder and heated up in a Joule-heated Knudsen cell. A K-type thermocouple connected to the crucible has been employed for measuring the ramp temperature during the warming up stage. The apparatus has been equipped with a cold cathode and a quartz crystal microbalance (QCM) placed in front of the source, thus allowing the real-time monitoring of both the pressure variations and the thickness of the deposited film. QCM signal and pressure trend during evaporation coupled with a preliminary AFM thickness calibration (see SI) have been used to monitor the deposition rate also for in situ experiments at synchrotron facilities.

The thermal evaporation of layers of $\mathrm{TbPc}_{2}$ on $\mathrm{Au}(111)$ single crystals was done directly at the ID08 beamline of ESRF, and in situ characterized by the X-ray natural linear dichroism (XLND) and X-ray magnetic circular dichroism (XMCD) measurements. The thermal evaporation of layers of $\mathrm{TbPC}_{2}$ on a polycrystalline $\mathrm{Au}$ film was performed at the SIM beamline of the SLS and in situ characterized (XMCD measurements).

ToF-SIMS characterization: ToF-SIMS analysis was carried out with a TRIFT III time-of-flight secondary ion mass spectrometer (Physical Electronics, Chanhassen, MN, USA) equipped with a gold liquid-metal primary ion source. Positive and negative ion spectra were acquired with a pulsed, bunched $22 \mathrm{keV} \mathrm{Au}^{+}$primary ion beam by rastering the ion beam over a $100 \mu \mathrm{m} \times 100 \mu \mathrm{m}$ sample area.

Analysis of the $X$-ray spectra: The $X$-ray absorption spectra of the $M_{4,5}$ edges of $\mathrm{Tb}^{3+}$ have been calculated within the ligand field multiplet approach with the Slater integrals associated to the Coulomb repulsions scaled down to $80 \%$ of the atomic values and the spin-orbit parameters shell $\zeta_{3 \mathrm{~d}}=13.368 \mathrm{eV}$ for the $3 d$ and $\zeta_{4 \mathrm{f}}=0.221 \mathrm{eV}$ for the $4 f$ shell. The spectra are linear combinations of three independent spectra $s_{0}, s_{1}$, and $s_{2}$ theshapes of which do not depend on the crystal field. We then did not consider any crystal field parameter for the calculation. In doing so one gets rid of numerous unknown parameters and focus on the two parameters that can be determined experimentally, that are [i $\left(\varepsilon \times \varepsilon^{*}\right)$ $<\mathrm{gs}|\mathrm{J}| \mathrm{gs}\rangle]$ given by XMCD and $\left[1 / 2<\mathrm{gs} \mid(\varepsilon \cdot \mathrm{J})\left(\varepsilon^{*} \cdot \mathrm{J}\right)+\left(\varepsilon^{*} \cdot \mathrm{J}\right)(\varepsilon \cdot\right.$ J) $\mid g s>-1 / 3 J(J+1)]$ given by XNLD. ${ }^{[19]}$

\section{Supporting Information}

Supporting Information is available from the Wiley Online Library or from the author. 


\section{Acknowledgements}

We acknowledge ESRF for providing beam time under the project HE3257 supported by the EC (FP7/2007-2013) under grant agreement n. ${ }^{\circ}$ 226716. The financial support from the EC (project MolSpinQIP, FP7-ICT-2007-C-211284), from Italian CNR (Commessa PM.P05.012), from Italian MIUR (PRIN2008FZK5AC and PRIN2008/858Y7 projects), from Italian MAE (program Italia-Polonia), and from Ente CRF is acknowledged.

Received: September 8, 2010 Published online: October 14, 2010

[1] C. Joachim, J. Gimzewski, A. Aviram, Nature 2000, 408, 541.

[2] L. Bogani, W. Wernsdorfer, Nature Mater. 2008, 7, 179.

[3] S. Sanvito, J. Mater. Chem. 2007, 17, 4455.

[4] D. Gatteschi, R. Sessoli, J. Villain, Molecular Nanomagnets; Oxford University Press, Oxford 2006.

[5] M. Mannini, F. Pineider, Ph. Sainctavit, C. Danieli, E. Otero, C. Sciancalepore, A. M. Talarico, M.-A. Arrio, A. Cornia, D. Gatteschi, R. Sessoli, Nature Mater. 2009, 8, 194.

[6] D. Gatteschi, A. Cornia, M. Mannini, R. Sessoli, Inorg. Chem. 2009, 48, 3408.

[7] M. Cavallini, M. Facchini, C. Albonetti, F. Biscarini, Phys. Chem. Chem. Phys. 2008, 10, 784.

[8] S. R. Forrest, Chem. Rev. 1997, 97, 1793.

[9] L. Margheriti, M. Mannini, L. Sorace, L. Gorini, D. Gatteschi, A. Caneschi, D. Chiappe, R. Moroni, F. Buatier de Mongeot, A. Cornia, F. M. Piras, A. Magnani, R. Sessoli, Small 2009, 5, 1460.

[10] L. Vitali, S. Fabris, A. M. Conte, S. Brink, M. Ruben, S. Baroni, K. Kern, Nano Letters 2008, 8, 3364

[11] K. Katoh, Y. Yoshida, M. Yamashita, H. Miyasaka, B. K. Breedlove, T. Kajiwara, S. Takaishi, N. Ishikawa, H. Isshiki,
Y. F. Zhang, T. Komeda, M. Yamagishi, J. Takeya, J. Am. Chem. Soc. 2009, 131, 9967.

[12] N. Ishikawa, Polyhedron 2007, 26, 2147.

[13] S. Stepanow, J. Honolka, P. Gambardella, L. Vitali, N. Abdurakhmanova, T.-C. Tseng, S. Rauschenbach, S-L. Tait, V. Sessi, S. Klyatskaya, M. Ruben, K. Kern, J. Am. Chem. Soc. 2010, $132,11900$.

[14] A. De Cian, M. Moussavi, J. Fischer, R. Weiss, Inorg. Chem. 1985 24, 3162.

[15] J. Souto, J. A. Desaja, R. Aroca, M. L. Rodriguez, Synth. Met. 1993 $54,229$.

[16] R. Jones, A. Krier, K. Davidson, Thin Solid Films 1997, 298 , 228

[17] J. B. Goedkoop, B.-T. Thole, G. Van Der Laan, G. A. Sawatzky, F. M. F. de Groot, J. C. Fuggle, Phys. Rev. B 1988, 37, 2086.

[18] B.-T. Thole, G. Van Der Laan, J. C. Fuggle, G. A. Sawatzky, R. C. Karnatak, J.-M. Esteva, Phys. Rev. B 1985, 32, 5107.

[19] J. P. Schillé, J.-P. Kappler, Ph. Sainctavit, Ch. Cartier dit Moulin, C. Brouder, G. Krill, Phys. Rev. B 1993, 48, 9491.

[20] H. Peisert, T. Schwieger, J. M. Auerhammer, M. Knupfer, M. S. Golden, J. Fink, P. R. Bressler, M. Mast, J. Appl. Phys. 2001, $90,466$.

[21] H. Peisert, I. Biswas, L. Zhang, M. Knupfer, M. Hanack, D. Dini, D. Batchelor, T. Chasse, Surf. Sci. 2006, 600, 4024.

[22] D. C. Qi, D. J. T. Sun, X. Y. Gao, L. Wang, S. Chen, K. P. Loh, A. T. S. Wee, Langmuir 2010, 26, 165.

[23] I. Biaswas, H. Peisert, M. Nagel, M. B. Casu, S. Schuppler, P. Nagel, E. Pellegri, T. Chassé, J. Chem. Phys. 2007, 126, 174704.

[24] I. Biaswas, H. Peisert, M. B. Casu, B.-E Schuster, P. Nagel, M. Merz, S. Schuppler, T. Chassé, Phys. Status Solidi A 2009, 206, 2524.

[25] M. Gonidec, E. S. Davies, J. Mcmaster, D. B. Amabilino, J. Veciana, J. Am. Chem. Soc. 2010, 132, 1756.

[26] I. Letard, Ph. Sainctavit, Ch. Cartier dit Moulin, J.-P. Kappler, P. Ghigna, D. Gatteschi, B. Doddi, J. Appl. Phys. 2007, 101, 113920. 